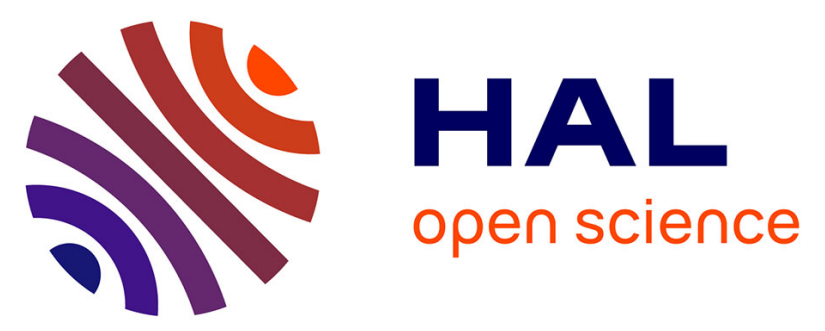

\title{
Les paysages et les hommes des Alpes du sud dans les carnets de Paul Vidal de La Blache
}

\author{
Roland Courtot
}

\section{To cite this version:}

Roland Courtot. Les paysages et les hommes des Alpes du sud dans les carnets de Paul Vidal de La Blache. Méditerranée, 2007, Les terroirs : caractérisation, développement territorial et gouvernance, 109, pp.9 - 15. 10.4000/mediterranee.104 . hal-03029593

\section{HAL Id: hal-03029593 \\ https://hal-amu.archives-ouvertes.fr/hal-03029593}

Submitted on 28 Nov 2020

HAL is a multi-disciplinary open access archive for the deposit and dissemination of scientific research documents, whether they are published or not. The documents may come from teaching and research institutions in France or abroad, or from public or private research centers.
L'archive ouverte pluridisciplinaire HAL, est destinée au dépôt et à la diffusion de documents scientifiques de niveau recherche, publiés ou non, émanant des établissements d'enseignement et de recherche français ou étrangers, des laboratoires publics ou privés.

\section{(ㅇ)(1) $\$$}

Distributed under a Creative Commons Attribution - NonCommercial - NoDerivatives 44.0 


\section{(2)enEdition Journals}

\section{Méditerranée}

Revue géographique des pays méditerranéens / Journal of Mediterranean geography

109 | 2007:

Terroirs : caractérisation, développement territorial et gouvernance

Du terroir vidalien à la gouvernance de terroir

\section{Les paysages et les hommes des Alpes du sud dans les carnets de Paul Vidal de La Blache}

Landscapes and peoples of the southern Alps in the notebooks of Paul Vidal de la Blache

\section{Roland COURTOT}

p. $9-15$

https://doi.org/10.4000/mediterranee.104

\section{Abstracts}

Français English

Avant de rédiger son « Tableau de la géographie de la France (Introduction à l'Histoire de France d'Ernest Lavisse, 1903) », Paul Vidal de la Blache s'est astreint à parcourir à la fin du 19e siècle, carnet à la main, le territoire français du nord au sud et de l'est à l'ouest. C'est ainsi qu'il a réalisé, entre 1888 et 1907, 9 voyages en Provence, dont 6 ont concerné les Alpes du sud, du Pelvoux aux Alpes-Maritimes et du col de Montgenèvre à la clue de Mirabeau. En partant de quelques paysages emblématiques décrits dans ses carnets de voyage, nous verrons comment il a privilégié l'étude des rapports entre l'homme et le milieu et comment il a posé les bases de ce qui serait plus tard l'analyse des terroirs.

Before writing his « Tableau de la géographie de la France (Introduction à l'histoire de France by Ernest Lavisse, 1903 ", Paul Vidalde la Blache, traveled up and down the territory of France, notebook in hand, from north to south and from east to west. Between 1888 and 1907, six of his nine trips to Provence were focused on the southern Alps from Pelvoux to the Maritime Alps and from Montgenèvre Pass to the opening of Mirabeau. Several emblematic landscapes described in Vidal's travel notebooks are examined here to show how he gave his major attention to the study

\section{This site uses cookies and collects personal data.}




\section{Full text}

\section{Introduction}

Paul Vidal de La Blache a parcouru à plusieurs reprises la Provence et les Alpes du sud au cours des voyages d'étude qu'il a entrepris pour réunir la documentation nécessaire à la rédaction du Tableau de la géographie de la France, premier volume de l'Histoire de France dirigée par Ernest Lavisse (1903 à 1922). Il n'est pas le premier géographe à s'intéresser aux Alpes du sud, comme l'a montré A. de RÉPARAZ (2000), mais il est certainement celui qui, avant R. Blanchard, a parcouru le premier scrupuleusement le terrain, crayon à la main, et avec un projet géographique précis et une méthode scientifique en cours d'élaboration. Les carnets de voyage qu'il a rédigés pendant ces itinéraires (carnets $\left.\mathrm{n}^{\circ} 11,12,14,15\right)$ nous ont permis d'identifier 8 voyages entre 1888 et 1907 . La carte qu'on peut en dresser montre que ses trajets sont sous la dépendance directe du seul moyen de communication rapide de son époque, le train. Par conséquent une partie des carnets est formée de notes prises au cours du voyage en train, ou le soir à l'étape... Il emprunte à plusieurs reprises les grands axes du PLM qui vient d'être prolongé jusqu'au Comté de Nice (vallée du Rhône et littoral provençal jusqu'à Menton) et l'axe secondaire de la Durance (en arrivant deux fois par le col de la Croix haute depuis Grenoble et une fois par le col du Montgenèvre après avoir débarqué à Oulx du train de Chambéryà Turin). Il utilise aussi les lignes secondaires et les chemins de fer locaux, dans la mesure de leur achèvement : ainsi le train de Nice à Digne, la voie de Grasse à Meyrargues par la Provence intérieure, la voie d'Avignon à Manosque, celle de Cavaillon à Pertuis. Voyageur pressé qui emprunte le chemin de fer chaque fois que possible (et note scrupuleusement l'état du réseau en cours de construction dans les Alpes du sud, voir carnet 15, folio 73), car il lui permet de parcourir de longues distances en observant le paysage depuis la fenêtre du wagon, comme un film documentaire continu qu'il observe au filtre de sa pensée géographique.

Mais il n'hésite pas aussi à parcourir du chemin en voiture et à pied pour explorer plus en détail les régions qui retiennent son attention, en particulier dans les montagnes et sur les littoraux. Le temps passé dans les différents lieux nous donne aussi une indication de ses centres d'intérêt, qui font la part belle à la Provence littorale entre Arles et Toulon, à la région de Nice et de Grasse, aux hautes vallées alpines (Briançonnais, Vallouise). Nous nous proposons donc de lire dans ces carnets la façon dont Vidal a envisagé les paysages des Alpes du sud à travers ses notes de terrain et comment celles-ci ont à la fois enrichi sa réflexion géographique et marqué les connaissances de la géographie des Alpes et de la Provence. Il ne s'agit pas de faire un inventaire complet des paysages étudiés, ni une étude épistémologique des carnets et du Tableau de la géographie de la France auquel ils ont servi de socle, ceux-ci ont déjà été faits (Robic et al., 2000). Il convient, en revanche, de retenir quelques exemples particulièrement développés parle géographe pour confronter ses conceptions géographiques au concept de terroir qui nous occupe aujourd'hui. Pour cela nous retiendrons trois paysages : les paysages de la haute montagne dans la Vallouise, ceux de la moyenne montagne sèche entre Digne et Entrevaux, et ceux du rebord des AlpesMaritimes. 


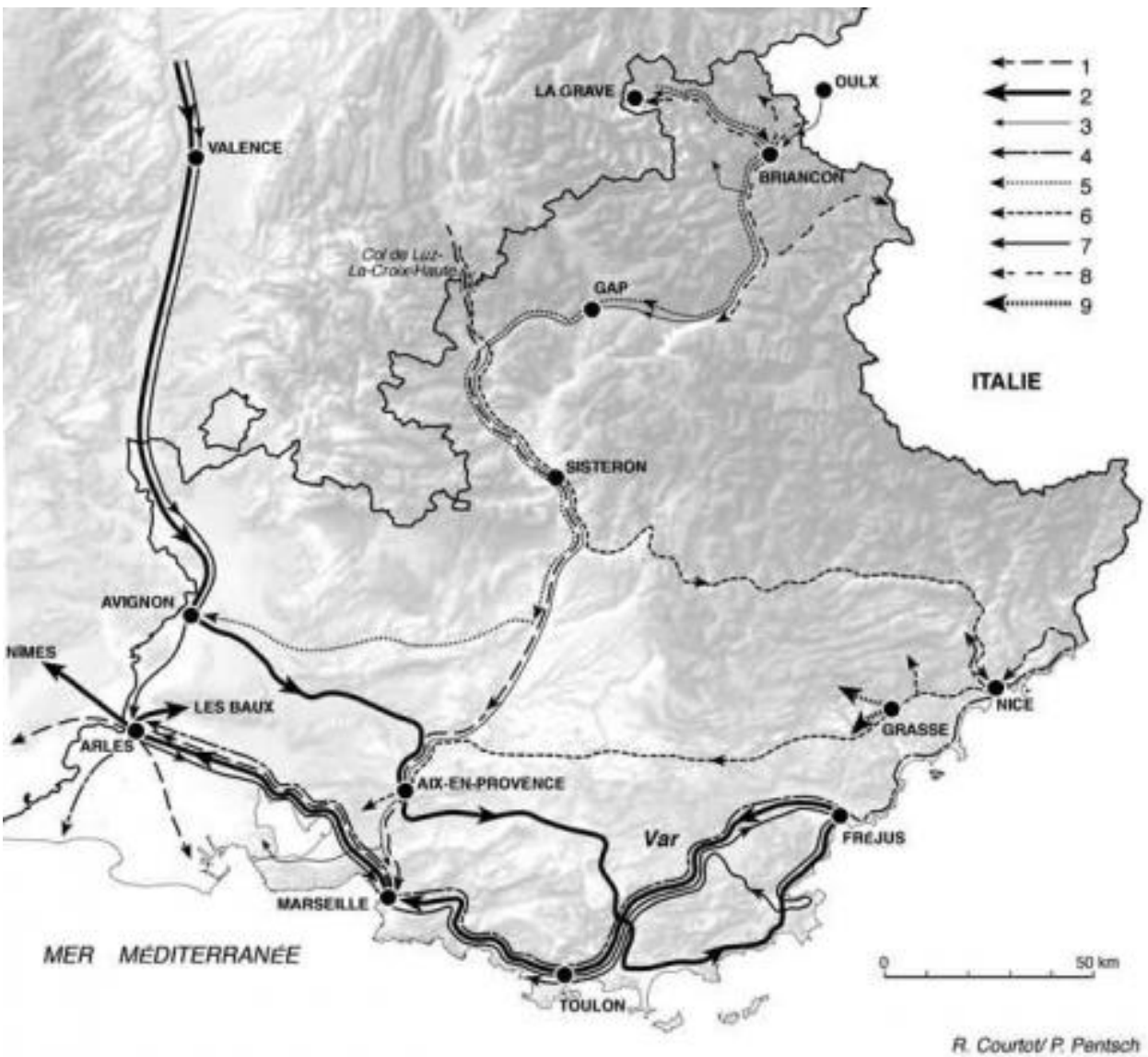

1 : Col de la Croix haute-Camargue par le val de Durance (carnet 11, p.85-95), sept.1888

2 : Orange-Arles par le Vaucluse, les Maures, Toulon (carnet 11, p.117-134 et 12, p.1.14) avril 1889

3 : Montgenèvre-Marseille par La Grave, Vallouise, Sisteron (carnet 12, p.32-65) sept.1889

4 : Nice-Camargue par Grasse et le littoral (carnet 12, p.181-200), mars 1899

5 : Col de la Croix haute-Cavaillon par le val de Durance (carnet 14, p.144-148) sept. 1892

6 : Col de la Croix haute, Digne, Nice, Grasse, Aix-en-Pce (carnet 15, p.52-77) avril 1892

7 : Lyon, Marseille, les Maures, Toulon (carnet 15, p.116-125) mars 1894

8 : La Grave, Queyras, Montgenèvre, Briançon (carnet 15, p.178-195), sept.1894

9 : Grasse, Cabris, St Cézaire (carnet 26, p.52-60), avril 1907.

\subsection{En Vallouise (carnet $n^{\circ} 12$, folio 46 à 57 )}

Lors d'un voyage en septembre 1889, arrivé à Oulx(Italie) par le train, il explore le col de Montgenèvre et celui du Lautaret, la région de la Grave, la vallée de Névache et celle de la Bérarde, puis par Briançon gagne la Vallouise, où il passe plusieurs jours, afin de visiter le pré de Mme Carle et de faire l'ascension de la Pointe de l'Aiglière $(3308 \mathrm{~m})$. Dans la haute montagne, il observe l'adaptation au milieu, au froid, l'isolement de la société, l'âpreté des genres de vie dans ce qu'il appelle, par rapport au massif du Pelvoux, "le versant des pierres, opposé au versant des pâtures » (celui-ci étant le versant de la Grave, où la désagrégation des roches est pour lui plus importante). Il note l'évolution de la vallée de l'amont vers l'aval:» jolie vallée transversale de l'Aile froide aux Claux, tranchée profonde du torrent (....) rochers calcaires sur la rive droite, sources, ruisseaux et irrigation au fond de la vallée, pleine d'arbres, de jardins, de cultures. Les maisons se succèdent sans interruption, villages à la file. Au contraire, au sud de Vallouise, villages à mi-côte sur des versants boisés et cultivés " (folio 49 ter). Il s'agit de la commune de Puy-Saint-Vincent, située sur un ubac. Il constate surtout la

This site uses cookies and collects personal data.

For further information, please read our Privacy Policy (updated on June 25, 2018). By continuing to browse this website, you accept the use of cookies.

\section{Close}


Pelvoux dans un ciel bleu de plomb, miroitement de l'air sur les cailloux en plein midi, désolation profonde » (folio 49 bis), et bien géographiques : " une mer de montagnes, une solitude de cimes, dans les plis desquelles rien n'indique qu'il y ait des hommes, des champs, des villes » (folio 54).

\subsection{La moyenne montagne sèche entre Bléone et Var (carnet 15, folio 54 à 63)}

Au printemps 1892, venant de Grenoble par chemin de fer, il abandonne la vallée de la Durance à Château-Arnoux et gagne Nice par Barrème, Annot et Entrevaux : certainement par la route nationale 85 (qui n'est pas encore la route Napoléon !) car le chemin de fer ne va que jusqu'à Mezel (l'ouverture jusqu'à Saint-André étant prévue pour le 1er mai de cette même année 1892). Il parcourt donc rapidement (en deux jours) ces «montagnes arides et décharnées encombrées d'éboulis ». Il a le temps de voir les « petits prés avec fruitiers et jardins » dans la basse vallée de la Bléone et d'en faire un croquis (fig.2) et s'intéresse ensuite beaucoup plus à la géomorphologie de ces " plissements extraordinaires de couches » et de ces " débris pierreux ou schisteux » qui « encombrent et recouvrent aux trois quarts les pentes », qu'à la population de ces « hameau(x) misérablement délabré(s) » ou de ces villes dont certaines rues sont associées à un «idéal de saleté ». Il note cependant au passage les «plantations de pins, après le col de Vergons ", des cultures de vignes en gradins dans le bassin d'Annot, et guette « entre Scaffarels et le Var, (la) réapparition des oliviers ».

Fig. 2 - Le versant nord de la vallée de la Bléone à l'aval de Digne

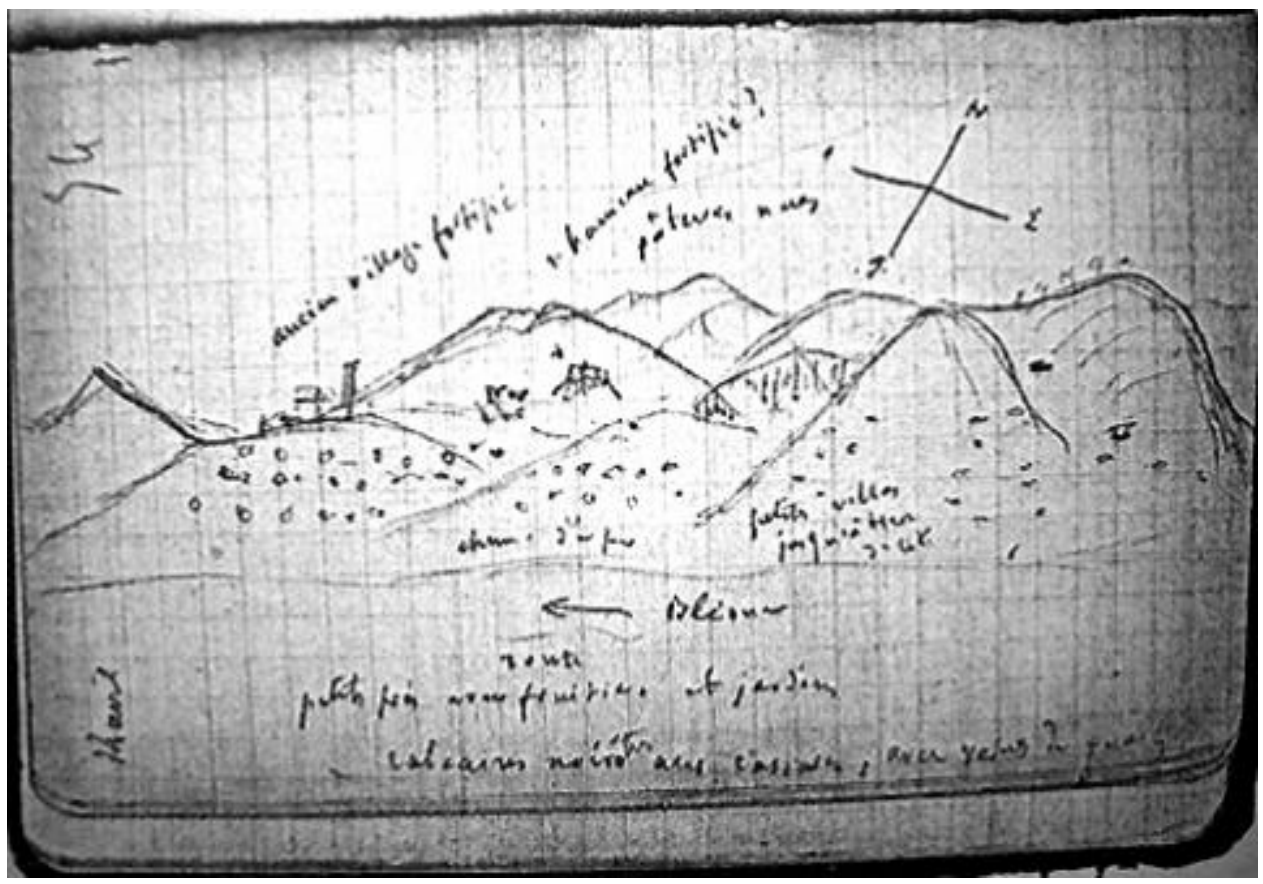

(Vidal de La Blache, carnet n`15, p.54, 14 avril 1892, @ Bibliothèque de l'Institut de Géographie de Paris). L'» ancien village fortifié » est celui des Hautes Sieyes, le " hameau fortifié » est celui de Courbons, les arbres qui couvrent les pentes sont des oliviers, les « petites villas jusqu'à tiers de côte » appartiennent à des habitants de Digne, et les sommets dont les pentes sont couvertes de " pâtures nues » sont, de gauche à droite : le Pic d'Oise, la Clapière haute, le Martignon (1430) et l'Andran. Au premier plan, les « calcaires noirâtres avec cassures, avec veines de quartz ", des " petits prés avec fruitiers et jardins ", la " route ", la " Bléone » et le « chemin de fer » sur la rive droite.

This site uses cookies and collects personal data.

For further information, please read our Privacy Policy (updated on June 25, 2018). By continuing to browse this website, you accept the use of cookies. 
25 - avril 1907, folio 52 à 60). Les trajets à pied doivent être importants et permettent une observation détaillée des faits géographiques, autant physiques qu'humains.

Sur le littoral à l'est de Nice, il déchiffre la géologie des " promontoires » et des « pitons aigus » et analyse les rapports étroits entre milieu physique et aménagements agraires : "Sans ces synclinaux crétacés, le pays serait inhabitable. (...) dès que les roches marneuses (...) apparaissent, le sol se couvre de cultures en terrasses (...).Au dessus, des maisons se montrent, tandis que, pour économiser le sol utile, les sentiers pierreux se tiennent sur le jurassique ». De même, les villages " posés en nids d'aigle au bord de précipices » retiennent son attention : «étroitement ramassé, entouré d'un bois d'olivier et de cultures en terrasses (...) dans le village, passages voûtés, portes cintrées ». La photographie du village de Peille, qu'il insère dans la version illustrée du Tableau (VidAL DE LA BLACHE, 1908) s'accompagne d'une légende qui reprend certaines notations du carnet : " Parfait type d'établissement méditerranéen, le bourg ne fait qu'un avec le jardin et le bois d'olivier, sorte d'oasis entre les roches. L'enceinte triangulaire se termine au sommet par un reste de casbah ou citadelle. Les maisons (...) subsistent en groupes serrés, et l'on circule entre elles par des passages voûtés ». Il développe donc une dialectique roches dures/roches tendres pour ces sites d'habitats et de cultures, accrochés aux pentes escarpées des Préalpes de Nice : ce sont des microterroirs agronomiques définis par la géologie des lieux.

La dernière excursion provençale concerne le rebord des Préalpes de Grasse entre Cabris et Saint-Vallier de Thieys. Relatée sous forme de croquis panoramiques abondamment légendés dans le carnet 26, elle prouve la maîtrise de l'observation géographique de Vidal sur le terrain. Le folio 53 est un excellent exemple d'analyse des terroirs agronomiques qui occupent les grands versants sous le village perché de Cabris (fig.4). Il note ainsi de bas en haut, du piémont vers la montagne:

- la prédominance des oliviers « jusqu'au fond de la vallée », soit en « bois », soit en terrasses, et qui montent jusqu'au premier front topographique calcaire du chevauchement ;

- l'occupation des pentes fortes par des « lambeaux de bois » («taillis de chênes blancs », « taillis mêlés de chênes verts et blancs », « quelques genévriers »);

- la présence de terrasses de cultures sur les versants encadrant les villages perchés (Cabris, Spéracèdes);

- les plateaux de calcaire arides, de type causse, déserts de pierre, déserts calcaires, au delà des corniches du rebord et autour des bassins montagnards (SaintVallier).

Étagements de cultures, limites biogéographiques et de l'habitat, contacts de mise en valeur et de densité, l'essentiel est dit : le géographe observe l'étagement altitudinal et le passage du milieu agricole du grand adret au milieu montagnard des causses calcaires. Il souligne en particulier, en passant le col de St-Vallier « partout, au voisinage du col, traces de cultures en gradins abandonnées » et un peu plus haut " quelques oliviers abandonnés sur un débris (?) de terrasses en gradins ». Les taillis de chênes et de buis cèdent la place aux pierrailles et la présence de moutons signale le passage à l'économie de l'élevage. Le bassin de St-Vallier est assimilé à un causse : " taillis de chênes ; quelques chênes très beaux ça et là. Cultures disséminées en gradins ; plus d'oliviers lignes simples- aspect de causse ».

Fig. 3 - Le village de Peille (photo G.J. fig. 266, in Le Tableau de la Géographie de la France, édition illustrée, Hachette, 1908). () Hachette

\section{Close}




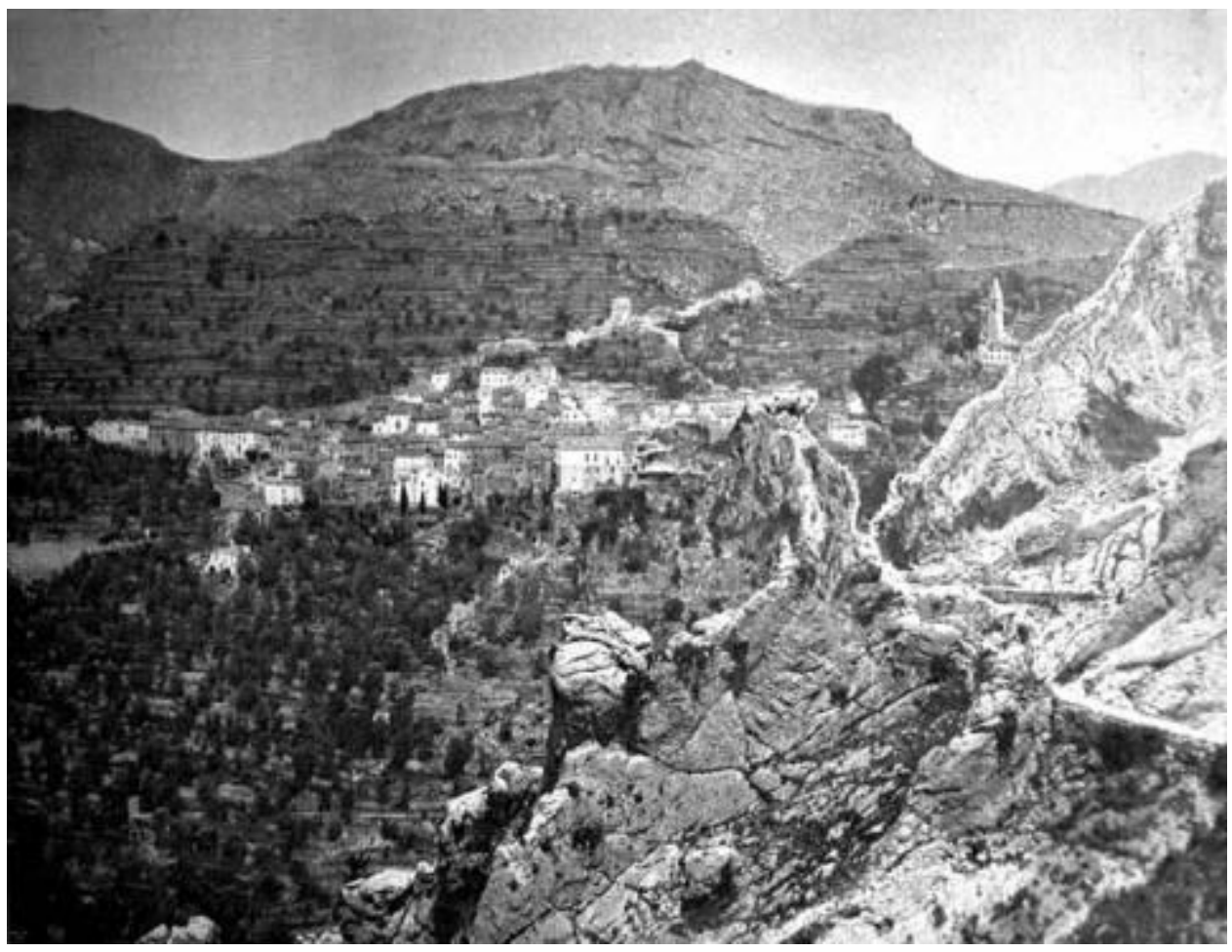

Sous le chapeau " Ossature de l'arrière-pays niçois, cultures et bourgs ", le village où Paul Vidal de La Blache a dû passer lors d'une excursion en 1899 est présenté comme le " parfait type d'établissement méditerranéen ».

\section{3 - L'homme et le milieu : de l'observation raisonnée à la géographie humaine}

On retrouve bien dans ces exemples la façon de voyager du géographe Paul Vidal de La Blache, tant attentif à recueillir des impressions esthétiques et sensibles du paysage qui l'entoure qu'à noter scrupuleusement les faits géographiques et leur évolution au fur et à mesure du déroulement de l'itinéraire. Ainsi, en descendant en train la vallée de la Durance, il écrit : "Sisteron, les oliviers se montrent, on va vers la lumière (...).Les montagnes se dépouillent ; garrigues au lieu des bois, villes ou bourgs sur des pitons rocheux, couleur grise des maisons se confondant presque avec la roche » (carnet 11, folio 83-84).

L'analyse paysagère met toujours en avant les faits physiques, les roches, les sols, la structure géologique, la végétation, avant de noter les faits agraires, les habitats, les habitants. Ce déterminisme a pu être reproché à Vidal. Il procède d'une démarche inductive sur le terrain, fortement marquée par le naturalisme (les sciences de la nature étaient alors, sur le terrain, des modèles à suivre), mais déjà tempérée par les nombreuses notations qui concernent les hommes et la civilisation dans les pages des carnets. Il est encore plus largement corrigé par la mise en avant du rôle de l'homme et de la dimension historique des sociétés quant on passe des carnets au Tableau de la Géographie de la France, puis aux Principes de Géographie humaine, pour devenir ce que les épistémologues de la géographie ont appelé le « possibilisme » vidalien. Ce n’est $1,1,, 1,1,1, \ldots, \ldots, 1, \ldots$ 
local, des rapports entre l'homme et le milieu suppose une connaissance des logiques du milieu naturel et des "systèmes productifs locaux » (pour utiliser un néologisme pratique) qui fait encore largement défaut, et qui sera l'œuvre des géographes de l'entredeux-guerres, dont les thèses de géographie régionale fourniront les bases géographiques et historiques de ce qu'on peut appeler aujourd'hui l'analyse des terroirs. On peut déjà noter, pour ce qui est du compte-rendu des observations de terrain dans le sud-est de la France, une différence remarquable entre les carnets qui ont précédé (et servi) la rédaction du Tableau, et celui de 1907qui concerne entre autres un voyage en Espagne (Соurтот, 2006) et les Préalpes de Grasse. Le format n'est plus celui d'un petit carnet d'écriture, où les croquis trouvent mal leur place, mais celui d'un carnet de dessin, où le texte est toujours présent, même pour signaler des entretiens sur le terrain, mais où le dessin prend une importance nouvelle, d'autant plus grande qu'on sait que Vidal n'a jamais eu la réputation, à l'inverse d'autres géographes, d'être un « bon »dessinateur. La comparaison entre les dessins des figures 2 et 4 illustre bien notre propos. Le premier n'est qu'un dessin topographique de localisation. Le second est une analyse de paysage comme on pourrait en trouver aujourd'hui dans les manuels d'analyse spatiale destinés aux étudiants de géographie, et qui définit par le dessin, mieux qu'un long texte, les différents éléments géographiques du paysage observés, les différents «terroirs » qui le composent. En ce sens, s'il n'est pas un bon dessinateur, Vidal est un dessinateur efficace. Ce qui n'est pas dit, c'est le fonctionnement précis du système socio-économique qui organisait ainsi le grand versant préalpin de Cabris : un travail pour les géographes à venir après lui.

Fig. 4 - Cabris et le rebord des Préalpes de Grasse, vue de la route de Saint-Vallier-deThiey (vue de l'est)

(Paul Vidal de La Blache, carnet n²6, p.53, avril 1907, ( ) Bibliothèque de l'Institut de Géographie de Paris)

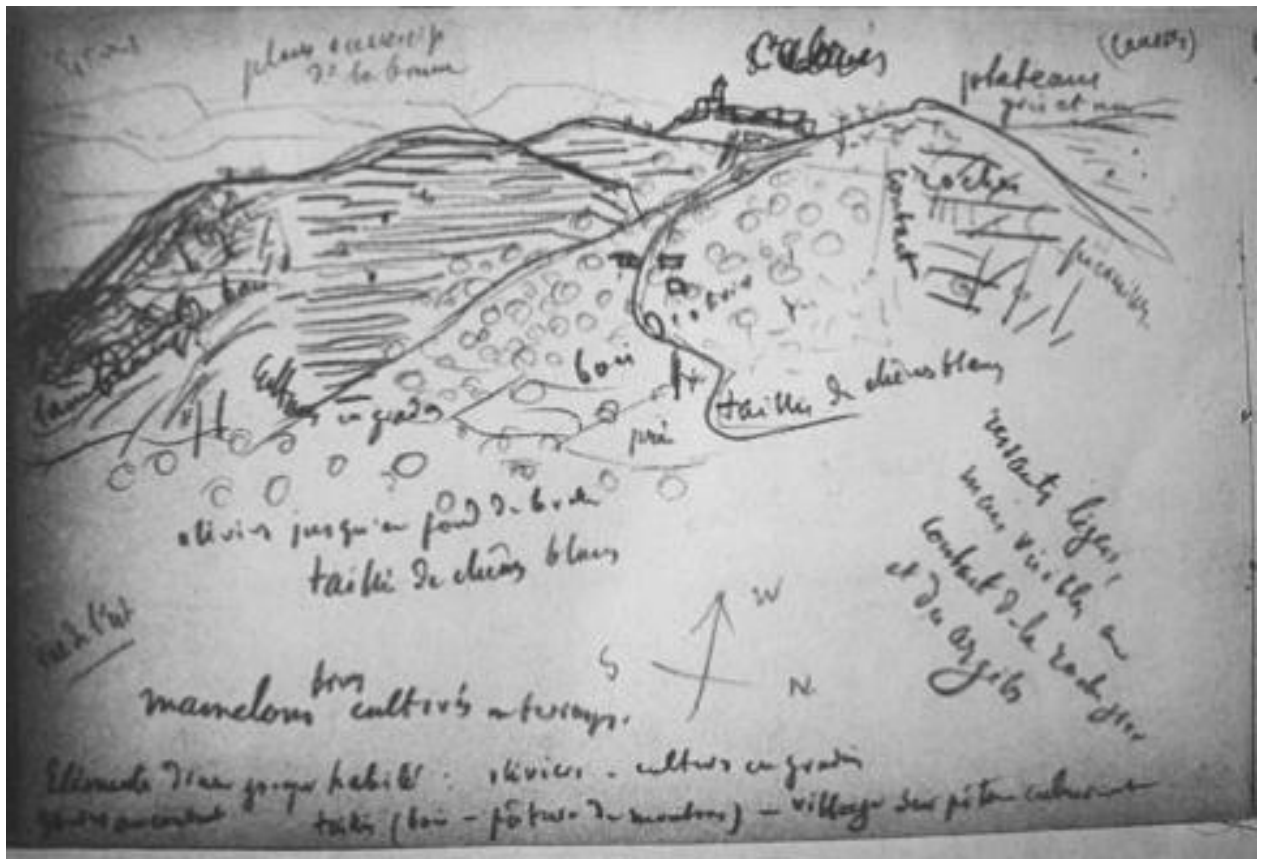

Légendes sur le croquis:

- à l'arrière plan gauche : Estérel, plans successifs dans la brume

- au centre : Cabris, à droite : plateaux gris et nus (causse)

- sur le versant à gauche : lambeaux de bois, cultures en gradins

- sur la pente de face : roches, pierrailles, contact / bois d'oliviers, taillis de chênes blancs, prés / oliviers jusqu'au fond de la vallée, taillis de chênes blancs

This site uses cookies and collects personal data.

For further information, please read our Privacy Policy (updated on June 25, 2018). By continuing to browse this website, you accept the use of cookies.

\section{Close}


11 Ces carnets géographiques, et parmi eux les pages étudiées sur les Alpes du sud, ont une double succession géographique. À travers la publication du tableau, ils fournissent une première approche "régionale »des Alpes du sud, même si la «personnalité géographique » de cet ensemble n'est pas clairement démontrée. Vidal traite des Alpes d'un côté et de la Provence de l'autre, sans définir exactement où s'arrêtent les premiers et où commence la seconde (ce qui d'ailleurs nous a permis de choisir librement nos exemples). Il est frappant de constater que ce voyage géographique produit et va diffuser sur la géographie des Alpes du sud, avec la caution d'un chef d'école, un certain nombre de connaissances qui auront valeur d'exemples : l'érosion, le déboisement, les déserts, la dépopulation, la sécheresse, la pauvreté, l’isolement. Ces thèmes ont été abordés avant lui (DE RÉPARAZ, 2000), mais ils vont passer à travers lui dans la recherche et l'enseignement universitaires. Les descriptions plus détaillées et exhaustives de Raoul Blanchard dans son œuvre monumentale sur les Alpes françaises reprendront souvent ces thèmes et la géographie scolaire emboitera le pas. Il faudra attendre les géographies régionales de la seconde moitié du XXe siècle pour que, sur les Alpes du sud, revisitées par des thèses et pénétrées par les axes de communication et le tourisme, soient forgées d'autres images, d'autres représentations, où les équilibres entre la nature et les hommes reposent sur de nouvelles bases. Pendant tout le XXe siècle, l'espace rural a été marqué, surtout dans la deuxième moitié du siècle, par l'introduction de moyens techniques qui ont permis un contrôle plus grand des contraintes du milieu physique (moyens mécaniques, chimiques...) et donc une intervention anthropique plus forte dans la « fabrication des terroirs », lesquels sont plus soumis aux logiques économiques des sociétés développées. On assiste donc à une artificialisation plus grande des terroirs et par contre coup des paysages ruraux. Dans le même temps, la société paysanne intègre les représentations patrimoniales et identitaires de ces complexes territoriaux comme valeur ajoutée des produits agricoles qui en sont issus. Dans les Alpes du sud, ce mouvement a été accompagné à l'inverse par celui de la rétraction et de l'abandon de certains terroirs condamnés par les contraintes physiques et économiques trop fortes.

Ces carnets ont participé à l'élaboration d'une géographie nouvelle, qui est devenue « classique » par la suite et critiquée comme telle. Mais cette géographie a fondé une école qui a marqué toute la première moitié du 20e siècle français. Au plan théorique, c'est la prise en compte de la profondeur historique de l'occupation humaine, des nuances de l'adaptation des sociétés à leur milieu, aux potentialités physiques que celuici leur offre (au plan des sols, du climat, de la végétation)... qui a jeté les bases du « possibilisme » de Vidal. Au plan pratique et méthodologique c'est la référence permanente au paysage, au terrain, au voyage, au paysage, qui a poussé le géographe sur les routes, le carnet et le crayon à la main. Celui-ci n'est plus, comme le géographe que rencontre le petit prince de Saint Exupéry, un dessinateur dans un bureau, qui cartographie les informations que lui fournit l'explorateur, mais quelqu'un qui est « attentif à des formes, des couleurs, des figures, des paysages ou des monuments, des tracés de routes.... », qui « doit focaliser sa capacité d'analyse, activer sa mémoire, mobiliser son imagination pour saisir, comprendre des sites particuliers, et des questions précises (Tissier J.-L.,in RoBic M.-C., 2000, p.25) », modèle qui a influencé par la suite des générations de géographes.

\section{Bibliography}

Claval P., (1993), Autour de Vidal de La Blache, Mémoires et Documents de Géographie, CNRS, I.nuic .Jean $150 \mathrm{n}$

This site uses cookies and collects personal data.

For further information, please read our Privacy Policy (updated on June 25, 2018). By continuing to browse this website, you accept the use of cookies.

\section{Close}


Vidal de la Blache P., (1908), La France, tableau géographique, Hachette, édition illustrée, Paris, $365 \mathrm{p}$.

Vidal de la Blache P. (1994), Tableau de la géographie de la France, La Table Ronde, Paris, (réédition du $1 \mathrm{er}$ volume de l'Histoire de la France depuis les origines jusqu'à la Révolution dirigée par Ernest Lavisse, Hachette, 1903-1922), 559 p.

\section{Notes}

1 Dans les archives de l'Institut de Géographie de Paris, une série de carnets de notes de terrain dont les " inventeurs » ont été Bernadette Joseph et Marie Dans les archives de l'Institut de Géographie de Paris, une série de carnets de notes de terrain dont les « inventeurs » ont été Bernadette Joseph et Marie-Claire Robic a été identifiée par cette dernière comme ceux que Vidal de la Blache a rédigés pendant ses nombreux voyages d'étude dans les pays européens, soit trentetrois carnets, qui s'étalent de 1867 à 1918, et qui concernent des régions françaises et plus d'une vingtaine de pays étrangers, surtout européens et voisins de la France (Courtot, 2006).

\section{List of illustrations}

\begin{tabular}{|c|c|c|}
\hline \multirow{2}{*}{\multicolumn{2}{|c|}{ Title }} & Fig. 1 - Itinéraires de Paul Vidal de La Blache en Provence \\
\hline & & $\begin{array}{l}1: \text { Col de la Croix haute-Camargue par le val de Durance (carnet 11, } \\
\text { p.85-95), sept.18882: Orange-Arles par le Vaucluse, les Maures, Toulon } \\
\text { (carnet 11, p.117-134 et 12, p.1.14) avril 18893: Montgenèvre-Marseille } \\
\text { par La Grave, Vallouise, Sisteron (carnet 12, p.32-65) sept.18894 : Nice- } \\
\text { Camargue par Grasse et le littoral (carnet 12, p.181-200), mars } 18995: \\
\text { Col de la Croix haute-Cavaillon par le val de Durance (carnet 14, p.144- } \\
\text { 148) sept. 18926: Col de la Croix haute, Digne, Nice, Grasse, Aix-en- } \\
\text { Pce (carnet 15, p.52-77) avril } 18927: \text { Lyon, Marseille, les Maures, Toulon } \\
\text { (carnet 15, p.116-125) mars 18948 : La Grave, Queyras, Montgenèvre, } \\
\text { Briançon (carnet 15, p.178-195), sept.18949: Grasse, Cabris, St Cézaire } \\
\text { (carnet 26, p.52-60), avril 1907. }\end{array}$ \\
\hline \multirow{3}{*}{\multicolumn{2}{|c|}{$\begin{array}{l}\text { URL } \\
\text { File } \\
\text { Title }\end{array}$}} & $\begin{array}{l}\text { http://journals.openedition.org/mediterranee/docannexe/image/104/img- } \\
\text { 1.jpg }\end{array}$ \\
\hline & & image/jpeg, 44k \\
\hline & & Fig. 2 - Le versant nord de la vallée de la Bléone à l'aval de Digne \\
\hline \multicolumn{2}{|l|}{ 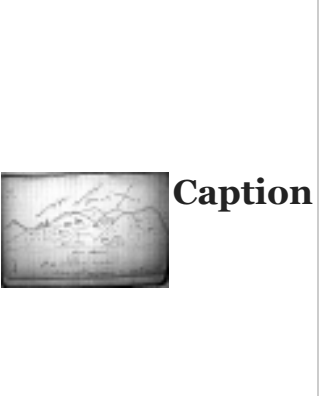 } & $\begin{array}{l}\text { (Vidal de La Blache, carnet n`15, p.54, } 14 \text { avril 1892, @ Bibliothèque de } \\
\text { l'Institut de Géographie de Paris).L'» ancien village fortifié » est celui des } \\
\text { Hautes Sieyes, le « hameau fortifié » est celui de Courbons, les arbres } \\
\text { qui couvrent les pentes sont des oliviers, les « petites villas jusqu'à tiers } \\
\text { de côte » appartiennent à des habitants de Digne, et les sommets dont } \\
\text { les pentes sont couvertes de « pâtures nues » sont, de gauche à droite: } \\
\text { le Pic d'Oise, la Clapière haute, le Martignon (1430) et l'Andran. Au } \\
\text { premier plan, les « calcaires noirâtres avec cassures, avec veines de } \\
\text { quartz », des " petits prés avec fruitiers et jardins », la « route », la } \\
\text { « Bléone » et le « chemin de fer » sur la rive droite. }\end{array}$ \\
\hline & UيL & $\begin{array}{l}\text { http://journals.openedition.org/mediterranee/docannexe/image/104/img- } \\
\text { 2.jpg }\end{array}$ \\
\hline & File & image/jpeg, 88k \\
\hline & Title & $\begin{array}{l}\text { Fig. } 3 \text { - Le village de Peille (photo G.J. fig. 266, in Le Tableau de la } \\
\text { Géographie de la France, édition illustrée, Hachette, 1908). (C Hachette }\end{array}$ \\
\hline \multirow[t]{2}{*}{ 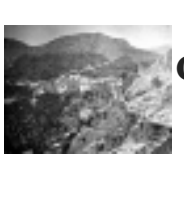 } & & $\begin{array}{l}\text { Sous le chapeau « Ossature de l'arrière-pays niçois, cultures et } \\
\text { bourgs », le village où Paul Vidal de La Blache a dû passer lors d'une } \\
\text { excursion en } 1899 \text { est présenté comme le « parfait type d'établissement } \\
\text { méditerranéen ». }\end{array}$ \\
\hline & & http://journals.openedition.org/mediterranee/docannexe/image/104/img- \\
\hline
\end{tabular}


chênes blancsAnnotations en marge:- à droite : ressauts légers mais visibles au contact de la roche grise et des argiles- au dessous : mamelons bruns cultivés en terrasses.Éléments d'un groupe habité situé au contact : oliviers-cultures en gradins, taillis (bois-pâture de moutons) village sur piton culminant.

URL

http://journals.openedition.org/mediterranee/docannexe/image/104/img4.jpg

File image/jpeg, 45k

\section{References}

Bibliographical reference

Roland Courtot, "Les paysages et les hommes des Alpes du sud dans les carnets de Paul Vidal de La Blache », Méditerranée, 109 | 2007, 9-15.

Electronic reference

Roland Courtot, «Les paysages et les hommes des Alpes du sud dans les carnets de Paul Vidal de La Blache », Méditerranée [Online], 109 | 2007, Online since 01 July 2009, connection on 28 November 2020. URL : http://journals.openedition.org/mediterranee/104 ; DOI : https://doi.org/10.4000/mediterranee.104

\section{This article is cited by}

- Prévost, Philippe. Capitaine, Mathieu. Gautier-Pelissier, François. Michelin, Yves. Jeanneaux, Philippe. Fort, Fatiha. Javelle, Aurélie. Moïti-Maïzi, Pascale. Lériche, Françoise. Brunschwig, Gilles. Fournier, Stéphane. Lapeyronie, Paul. Josien, Étienne. (2014) Le terroir, un concept pour l'action dans le développement des territoires. VertigO. DOI: 10.4000/vertigo.14807

- Hallair, Gaëlle. (2013) Les carnets de terrain du géographe français Emmanuel de Martonne (1873-1955) : méthode géographique, circulation des savoirs et processus de visualisation. Belgeo. DOI: 10.400o/belgeo.10807

\section{About the author}

\section{Roland Courtot}

Professeur émérite, UMR TELEMME MMSH - Université de Provence, Aix-en-Provence

By this author

Les paysages lus du ciel. De l'intérêt de la photographie aérienne oblique [Full text]

Landscapes from the sky, the interest of aerial photography

Published in Méditerranée, 120 | 2013

Le géographe et le tapis volant [Full text]

André HUMBERT, 2012, Casa de Velazquez, coll. Essais, vol. 5, Madrid, 188 p.

Published in Méditerranée, 120 | 2013

Pietro Laureano, Jardins de pierre. Les Sassi de Matera et la civilisation méditerranéenne [Full text]

(2005), Presses Universitaires de Vincennes, Saint-Denis, Coll. "Temps et Espaces", 172 p., (traduit de l'italien par Sidonie Joannès).

Published in Méditerranée, 107 | 2006

Évolution de l'occupation du sol en Provence-Alpes-Côte d'Azur vue du ciel [Full text] Analvse de l'évonlı ıtionn de l'nc.cı ınationn dı snl à nartir dẹs dnnnées C.ORINF I and Conver (1988) 